\title{
Effects of Probiotics, Prebiotics, and Synbiotics on Messenger RNA Expression of Caveolin-1, NOS, and Genes Regulating Oxidative Stress in the Terminal Ileum of Formula-Fed Neonatal Rats
}

\author{
ANTONI D'SOUZA, LAWRENCE FORDJOUR, ASMA AHMAD, CHARLES CAI, DHARMENDRA KUMAR, GLORIA VALENCIA, \\ JACOB V. ARANDA, AND KAY D. BEHARRY
}

Department of Pediatrics [A.D., L.F., A.A., C.C., D.K., G.V., J.V.A.], State University of New York, Downstate Medical Center, Brooklyn, New York 11203; Department of Pediatrics [K.D.B.], University of California, Irvine, Irvine, California 92868

\begin{abstract}
Necrotizing enterocolitis (NEC) afflicts extremely low birth weight neonates, and probiotics reduces its incidence and severity. NO is involved in the pathogenesis of NEC, and caveolin-1 regulates NO signaling. We tested the hypothesis that intestinal caveolin-1 and NOS are deficient in formula-fed neonatal rats and that supplementation with "Florastar Kids" and/or galactooligosaccharides and fructo-oligosaccharides preserves caveolin-1 and NOS. At birth (P0), neonatal rat pups were maternally fed or hand-gavaged with or without supplemented formula. Samples from the terminal ileum were analyzed for total NO metabolites, growth factors, and gene expression of caveolin-1, NOS isoforms, and antioxidants. Our data showed that formula feeding with and without supplementation resulted in significant growth restriction. Despite suboptimal nutrition, growth factors involved in intestinal repair and regeneration were increased in the neonatal rat ileum. Caveolin-1, endothelial NOS, and neuronal NOS were simultaneously downregulated with formula feeding while inducible NOS was upregulated. Superoxide dismutase and glutathione peroxidase were up-regulated with supplementation. These data provide a probable mechanism for the benefits of supplemented formula for decreasing the severity of NEC by preserving the antioxidant systems. (Pediatr Res 67: 526-531, 2010)
\end{abstract}

$\mathrm{N}$ ecrotizing enterocolitis (NEC) occurs predominantly in $\sim 12 \%$ of infants born with birth weights $<1500 \mathrm{~g}$, approximately one third of whom succumb to the disease (1). Loss of epithelial integrity, marked inflammation, and tissue necrosis are the hallmarks of NEC. The role of NO in pathophysiology of NEC is well known. NO is an extremely soluble, highly reactive free radical that is formed by the conversion of arginine to citrulline by NOS, for which three isoforms exist (2) namely, the constitutional neuronal isoform (NOS I or nNOS), the inducible isoform (NOS II or iNOS), and the endothelial isoform (NOS III or eNOS). Although both constitutive and inducible isoforms of NOS are present in

Received September 8, 2009; accepted January 8, 2010.

Correspondence: Antoni D'Souza, M.D., Division of Neonatal-Perinatal Medicine, Department of Pediatrics, State University of New York, Downstate Medical Center, 450 Clarkson Avenue, Box 49, Brooklyn, NY 11203; e-mail: ad'souza@downstate.edu

Supported by a grant from the Department of Pediatrics, SUNY Downstate Medical Center, Brooklyn, NY.

Presented, in part, at the Pediatric Academic Societies 2009 Annual Meeting, Baltimore, MD, May 2-5, 2009. the small intestines, $90 \%$ is nNOS (3-5). The iNOS is induced in inflammation and plays a role in the pathogenesis of gut barrier failure (6). NO reacts with superoxide $\left(\mathrm{O}_{2}{ }^{-}\right)$to produce the potent oxidant peroxynitrite (7), which is responsible for cytopathic damage (8). Superoxide dismutase (SOD) and glutathione peroxidase (GPX) enhance NO bioavailability by removing $\mathrm{O}_{2}^{-}$and preventing conversion of $\mathrm{NO}$ to reactive oxygen species (ROS) $(3,9)$.

Caveolin-1 is involved in regulating the functions of the different NOS isoforms. Caveolin-1, eNOS, nNOS, and iNOS have significant roles in inflammation, microvascular permeability, immunomodulation, cell growth, lipid trafficking, endocytosis, and cell migration (10-12). Caveolin-1 are also linked to the functions of many growth factors, including IGF-I, IGF-2, and EGF, which are present in high concentrations in the liquids swallowed by the fetus and neonate. These growth factors regulate intestinal epithelial barrier function, migration, and proliferation of enterocytes in response to mucosal injury; decrease intestinal apoptosis; down-regulate proinflammatory responses; and induce growth and development of the gastrointestinal tract (13). The receptors for these growth factors are expressed on enterocytes of the fetus and neonate, and caveolae are abundant in those receptors $(14,15)$. Low growth factors predispose the immature gut to the development of NEC. The use of probiotics has recently been shown to prevent NEC in extremely low birth weight (ELBW) infants (16). Prebiotics, fructo-oligosaccharides (FOS) and galacto-oligosaccharides (GOS), are nondigestible food ingredients that stimulate growth and activity of beneficial bacteria. Synbiotics, a combination of probiotics and prebiotics, which improves the survival of the probiotic organism and the intestinal environment, have also been shown to be beneficial. The effects of probiotics, prebiotics, and synbiotics on caveloin- 1 and NO signaling and on growth factors in the terminal ileum have not been studied.

Abbreviations: eNOS, endothelial NOS; FOS, fructo-oligosaccharides; GOS, galactoligosaccharides; GPX, glutathione peroxidase; iNOS, inducible NOS; NEC, necrotizing enterocolitis; nNOS, neuronal NO synthase; NOx, NO metabolites; $\mathbf{O}_{\mathbf{2}}{ }^{-}$, superoxide anion; ROS, reactive oxygen species; SOD, superoxide dismutase 
Formula-fed rats exhibit various degrees of inflammatory changes and share many histopathologic and clinical features of NEC seen in ELBW infants (17-19). Therefore, by using the rat model, we tested the hypothesis that intestinal caveolin-1 and NOS are deficient in formula-fed neonatal rats and that supplementation preserves caveolin-1 and NOS signaling. Because caveolin-1 is regulated by IGF-I, and IGF-I receptors are enriched with caveolae (20), our first objective was to examine the effects of enteral feeding with and without supplementation on IGFs and EGF in the neonatal rat terminal ileum. Furthermore, because SOD and GPX enhances NO bioavailability by removing $\mathrm{O}_{2}{ }^{-}$thereby preventing the formation of ROS $(3,9)$ and decreasing caveolin-1 levels, our secondary objective was to establish whether the mechanisms involved in the benefits of supplementation with probiotics, prebiotics, or synbiotics involves ROS scavengers.

\section{MATERIALS AND METHODS}

Animals. This study was approved by the Animal Care and Use Committee of the State University of New York, Downstate Medical Center, Brooklyn, NY. Certified infection-free, timed-pregnant Sprague Dawley rats were purchased at $18 \mathrm{~d}$ gestation from Charles River Laboratories (Wilmington, MA). The animals were housed in a 12 -h day/12-h night cycle and were provided standard laboratory diet and water ad libitum. Within $24 \mathrm{~h}$ of birth, newborn pups delivered on the same day were pooled and randomly assigned to expanded litter sizes of 18 pups/litter to simulate relative postnatal malnutrition of critically ill ELBW infants. Each pup was weighed and measured for body length (crown to rump, $\mathrm{cm}$ ) before experimentation and every $24 \mathrm{~h}$.

Experimental design. Five groups, pooled from 10-12 litters were studied. Formula-fed newborn rat pups were separated from their mothers from the first day of life (P0) and kept at $37^{\circ} \mathrm{C}$ using a water-jacketed heating pad attached to a pump (Gaymar Industries, Orchard Park, NY). Group 1 received Similac Advance infant formula with iron, ready to feed, $20 \mathrm{cal} / \mathrm{fl} \mathrm{oz}$ with special nutrients found in breast milk and used as an alternative to breast milk from Ross/Abbot Laboratories (Form-Fed; Abbott Park, IL). Group 2 received Similac Advance infant formula with iron supplemented with Florastor kids, the \# 1 Probiotic worldwide made of live, freeze-dried yeast cells of the species Saccharomyces Boulardii lyo (S. Boulardii, $5 \mathrm{mg} / \mathrm{mL}$ ) from Biocodex, Inc. San Bruno, CA (Pro-Fed). Group 3 received Similac Advance Early Sheild with iron, ready to feed, $20 \mathrm{cal} / \mathrm{fl} \mathrm{oz}$ with prebiotics (GOS/FOS) from Ross/Abbott Laboratories (Pre-Fed). Group 4 received Similac Advance Early Shield supplemented with $5 \mathrm{mg} / \mathrm{mL} S$. Boulardii and FOS/GOS or synbiotics (Syn-Fed). The doses of $S$. Boulardii and GOS/FOS used in our study were consistent with the manufacturer's recommendations for use in children. Group 5 (control) was maternally fed (Mat-Fed) using the same dam throughout the study. The formula-fed groups (groups 1-4) were administered with 0.2 - $\mathrm{mL}$ formula every $3 \mathrm{~h}$, including nighttime, using sterile $1-\mathrm{mL}$ syringes attached to specialized feeding needles (Harvard Apparatus, Inc. Holliston, MA) from P0 to P3 before killing. Each group had a separate feeding needle. After each feeding, the needles were washed, resterilized, and stored in labeled, sealed, sterile conical tubes until the next feeding time. The 1-mL syringes were discarded after each feeding. The pups were weighed and measured daily for weight loss or gain.

Sample collection. On $\mathrm{P} 4$, the surviving pups were killed by decapitation and samples from the terminal ileum were collected for histologic, biochemical, and molecular analyses. To identify the terminal ileum, the intestines were dissected out and biopsies were obtained from the most terminal area. For light microscopy, $2-3$ biopsies $(\sim 1 \mathrm{~cm})$ were obtained from four representative pups in each group. The specimens were placed in $10 \%$ neutralbuffered formalin and taken to the Pathology Department for processing using graded alcohols and paraffin embedding. For analysis of growth factors, fresh tissue samples $(200 \mathrm{mg})$ were rinsed in ice-cold PBS to remove blood elements and then placed in tubes containing ceramic beads and $1.0-\mathrm{mL}$ sterile normal saline. The samples were homogenized using a Fast-Prep instrument (MP Biomedicals, Solon, $\mathrm{OH}$ ). The homogenates were centrifuged at $8000 \mathrm{rpm}$ for $20 \mathrm{~min}$ at $4^{\circ} \mathrm{C}$ and filtered before assay for IGFs, EGF, and NO stable metabolites (NOx). Levels in the samples were standardized using total cellular protein levels. For mRNA extraction, fresh tissue samples $(\sim 100$ $\mathrm{mg}$ ) were rinsed in ice-cold PBS and placed nuclease-free microtubes and frozen immediately at $-70^{\circ} \mathrm{C}$ until assay.
Histology of the terminal ileum. Five-micrometer sections, cut from the paraffin blocks and mounted on superfrost slides, were stained with Harris hematoxylin and phloxin B. Image analysis was performed using Spot RT3 software (Diagnostic Instruments, Inc., Sterling Heights, MI), in combination with a high-resolution camera (Diagnostic Instruments, Inc., Sterling Heights, MI), Nikon Microphot-SA microscope (Nikon, Torrance, CA), and a Macintosh G4 Computer (Apple, Cupertino, CA). Images were digitized at $640 \times$ 480 pixels and $10 \times$ magnification. Histopathologic and morphometric analyses were conducted in a blinded manner. Morphometric analyses included ileal circumference $(\mu \mathrm{m})$, number of villi, and wall thickness $(\mu \mathrm{m})$. Wall thickness measurements were made at the 12,3,6, and 9 o' clock positions over the whole section. The number of villi was determined by dividing each section into four quadrants and counting the number in each quadrant. Five sections were analyzed for each group. For each section, there were four measurements of circumference, number of villi, and 12,3, 6, and 9 o' clock positions of wall thickness.

Analysis of IGFs. Active IGF-I and IGF-2 levels in the terminal ileum homogenates were determined using commercially available nonextraction mouse/rat enzyme-linked immunosorbent kits (Diagnostic Systems Labs), according to the manufacturer's protocol. Concentrations in the samples were calculated from a standard curve that ranged from 0 to $600 \mathrm{ng} / \mathrm{mL}$ for IGF-I and 0 to $2200 \mathrm{ng} / \mathrm{mL}$ for IGF-2. For IGF-I, the sensitivity of the assay was $0.015 \mathrm{ng} / \mathrm{mL}$, and the intra- and inter-assay coefficient of variation was $<12 \%$. For IGF-2, the sensitivity of the assay was $2.2 \mathrm{ng} / \mathrm{mL}$, and the intraand inter-assay coefficient of variation was $<10 \%$. Data from ileum homogenates were standardized using total cellular protein levels.

Assay of EGF. EGF levels in the terminal ileum homogenates were measured using a quantikine mouse immunoassay kit (R \& D Systems, Minneapolis, MN) according to the manufacturer's protocol. EGF concentrations were calculated from a standard curve ranging from 0 to $500 \mathrm{pg} / \mathrm{mL}$. The intra- and interassay coefficients of variations are $<10 \%$, and the sensitivity of the assay is $0.95 \mathrm{pg} / \mathrm{mL}$. Data from ileum homogenates were standardized using total cellular protein levels.

Assay of NOx. NOx levels in terminal ileum homogenates were measured using total NO assay kits (Assay Designs, Inc., Ann Arbor, MI). The kit quantitatively determines total NO in biologic fluids and involves the enzymatic conversion of nitrate to nitrite by enzyme nitrate reductase followed by a colorimetric detection of nitrite as a colored azo dye product of the Griess reaction. Concentrations in the samples were calculated from a standard curve ranging from 0 to $1000 \mu \mathrm{M}$. The intra- and inter-assay coefficients of variations are $<10 \%$, and the sensitivity of the assay is $0.625 \mu \mathrm{M}$. Data from ileum homogenates were standardized using total cellular protein levels.

Total cellular protein levels. Terminal ileum total cellular protein levels were determined using the dye-binding Bio-Rad protein assay (Bio-Rad, Hercules, CA) with BSA as standard. The standard curve is linear from 0.05 to $1.45 \mathrm{mg} / \mathrm{mL}$ of protein. The protein assay was done on the same day as the assays for growth factors.

Quantitative real-time PCR. Total RNA was extracted using RNA Pro solution (MP Bio, Solon, $\mathrm{OH}$ ) and allowed to digest for $5 \mathrm{~min}$ at room temperature. The samples were transferred to microtubes containing ceramic beads and placed in a FastPrep-24 instrument (MP Bio) for $40 \mathrm{~s}$. After addition of chloroform, the samples were vortexed for $30 \mathrm{~s}$ and centrifuged at $13,000 \mathrm{rpm}$ at $4{ }^{\circ} \mathrm{C}$ for $20 \mathrm{~min}$. The upper aqueous phase was transferred to a clean Eppendorf tube containing $0.5 \mathrm{~mL}$ of ice-cold $100 \%$ ethanol. The samples were placed in a $-20^{\circ} \mathrm{C}$ freezer overnight to precipitate the RNA. After precipitation, the samples were centrifuged at $13,000 \mathrm{rpm}$ at $4{ }^{\circ} \mathrm{C}$ for 20 min, and the resulting pellet was washed in $75 \%$ ethanol/water and redissolved in $100 \mu \mathrm{L}$ nuclease-free water. Cleanup of the RNA was performed using RNEasy mini cleanup kits (Qiagen, Valencia, CA). One microgram of total RNA was reversely transcribed with random primers according to the manufacturer's protocol. The cDNA was diluted and the real-time PCR was performed using RT ${ }^{2}$ Profiler Rat NO Signaling PCR Array System (SABiosciences, Frederick, MD). The real-time PCR arrays were done on 96-well plates precoated with 84 genes in the NO signaling pathway. An aliquot of 25 $\mu \mathrm{L}$ was added to each well. Five different control genes present on the PCR array plates were used to normalize the mRNA expression of the genes studied. Calculations were made by uploading the real-time amplification data into the SABiosciences RT ${ }^{2}$ Profiler PCR Array Data Analysis web portal. Quantitative PCR was based on the cycle threshold $(\mathrm{Ct})$ value. A gene was considered not detectable if the $\mathrm{Ct}$ value was $>32$.

Statistical analysis. One-way ANOVA was used to determine differences among the groups for normally distributed data, and Kruskal-Wallis test was used for nonnormally distributed data after Bartlett's test for equality of variances. Post hoc analysis was performed using the Tukey and StudentNewman-Keuls tests for significance. Significance was set at $p<0.05$, and data are reported as mean \pm SEM, where applicable. All analyses were two-tailed and performed using SPSS (SPSS, Inc., Chicago, IL). 


\section{RESULTS}

Effect on growth. Randomization at birth resulted in comparable mean total body weight and body length in all groups. All animals survived at P1; however, mean body weight were significantly lower in the Form-Fed $(5.9 \pm 0.09, p<0.01)$, Pro-Fed $(6.0 \pm 0.08, p<0.01)$, Pre-Fed $(5.9 \pm 0.09)$, and Syn-Fed $(6.0 \pm 0.15, p<0.01)$ groups compared with Mat-Fed $(7.74 \pm 0.10)$. Similarly, mean linear growth was suppressed in the Form-Fed, Pro-Fed, Pre-Fed, and Syn-Fed groups $(4.0 \pm 0.09,4.1 \pm 0.04,4.2 \pm 0.04$, and $4.1 \pm 0.06$, respectively; $p<0.01)$ compared with Mat-Fed (4.6 \pm 0.04$)$. By postnatal d 2 and 3, the mortality rate was higher in the Form-Fed group compared with the supplemented formula groups, but the differences were not statistically significant. By postnatal $\mathrm{d} 4$, mortality rate was $55 \%(n=10)$ in the Form-Fed group, $72 \%(n=13)$ in the Pro-Fed group, and $66 \%(n=12)$ in the Pre-Fed and Syn-Fed groups. The survival rate was $100 \%(n=18)$ in the Mat-Fed group. Only the pups that survived on the P4 were used for the analysis, and they were killed by decapitation. No samples were collected after spontaneous expiration for analysis. Weight loss continued to be significantly higher in all formula-fed groups on $\mathrm{P} 2(5.0 \pm 0.10,4.8 \pm 0.09,5.1 \pm 0.10$, and $6.0 \pm 0.15$, respectively; $p<0.001)$ and $\mathrm{P} 3(4.3 \pm 0.10,4.1 \pm 0.04$, $4.3 \pm 0.07$, and $4.8 \pm 0.19$, respectively; $p<0.001)$ compared with Mat-Fed on P2 (8.4 \pm 0.06$)$ and P3 (9.1 \pm 0.10$)$. Mean linear growth remained lower on P2 $(4.1 \pm 0.07,4.0 \pm$ $0.04,4.2 \pm 0.05$, and $4.3 \pm 0.06$, respectively; $p<0.01)$ compared with Mat-Fed (4.7 \pm 0.06$)$ and on P3 (4.1 \pm 0.03 , $4.3 \pm 0.04,4.2 \pm 0.05$, and $4.3 \pm 0.11$, respectively; $p<$ $0.01)$ compared with Mat-Fed (4.9 \pm 0.06 ; Figs. 1and 2).

IGF-1, IGF-2, EGF, and NOx levels. IGF-1, IGF-2, and EGF levels in the terminal ileum homogenates from rat pups at P4 are shown in Figures 3-5. IGF-1 was elevated in all supplemental groups, but none reached statistical significance. IGF-2 levels (ng/mg protein) were significantly elevated in the prebiotics $(19.5 \pm 1.40, p<0.05)$ and synbiotic $(17.0 \pm 3.10$,

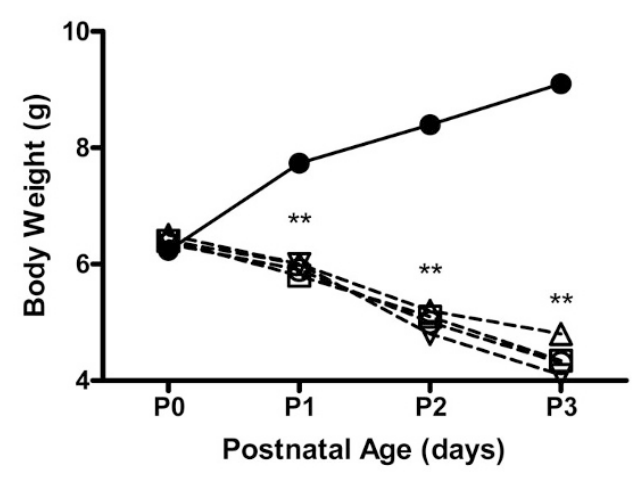

Figure 1. Changes in body weight in maternally fed (Mat-Fed, $\bullet$ ) neonatal rats and unsupplemented formula-fed rats (Form-Fed, $\bigcirc$ ); formula-fed rats supplemented with "Florastar Kids" (Pro-Fed, $\nabla$ ); formula-fed rats supplemented with GOS/FOS (Pre-Fed, y); and formula supplemented with combined "Florastar Kids" and GOS/FOS (Syn-Fed, $\triangle$ ). $n=18$ pups/group for all groups on P0 and P1. On P3, $n=18$ in Mat-Fed group; $n=10$ in Form-Fed group; $n=13$ in Pro-Fed group; $n=12$ in Pre-Fed group; and $n=12$ in Syn-Fed group. ${ }^{* *} p<0.01$ vs Mat-Fed.

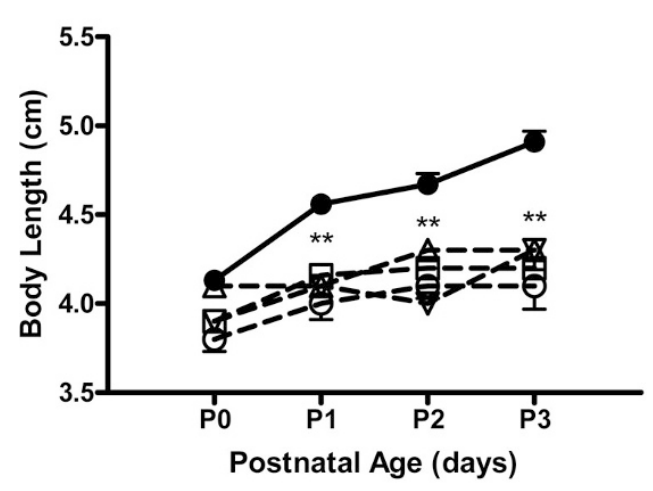

Figure 2. Changes in body length $(\mathrm{cm})$. Groups are as described in Figure. 1. $* * p<0.01$ vs Mat-Fed.

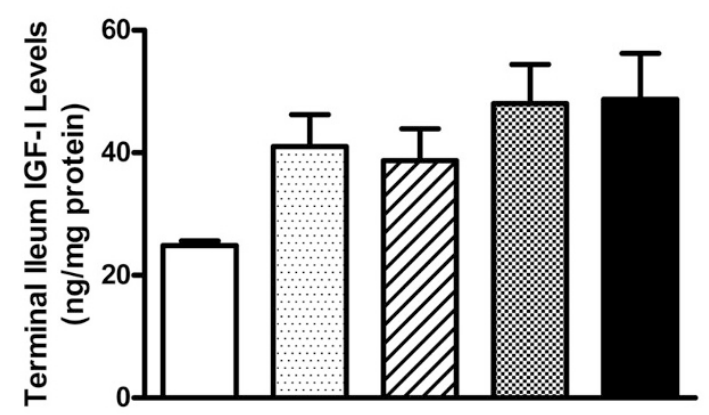

Figure 3. Mean IGF-1 levels (ng/mg of protein) in terminal ileum homogenates. Groups are as described in Figure 1. Open bar, Mat-Fed; lightly shaded bar, Form-Fed; lined bar, Pre-Fed; heavily shaded bar, Pro-Fed; and solid bar, Syn-Fed. Only the pups that survived on the P4 were used for the analysis.

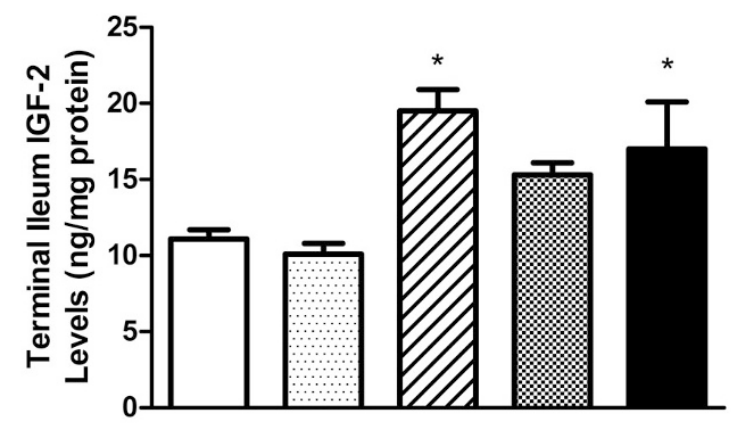

Figure 4. Mean IGF-2 levels (ng/mg of protein) in terminal ileum homogenates. Groups are as described in Figure 1. Open bar, Mat-Fed; lightly shaded bar, Form-Fed; lined bar, Pre-Fed; heavily shaded bar, Pro-Fed; and solid bar, Syn-Fed. Only the pups that survived on the P4 were used for the analysis. $* p<0.05$ vs Mat-Fed.

$p<0.05)$ groups compared with the maternally fed group $(11.1 \pm 0.60)$. EGF levels ( $\mathrm{ng} / \mathrm{mg}$ protein) were elevated in all supplemental groups and significantly increased in the formula-fed $(1.2 \pm 0.31, p<0.05)$ and probiotic-fed $(1.2 \pm 0.33$, $p<0.05)$ groups compared with the maternally fed group $(0.4 \pm 0.01)$. NOx levels were low or undetectable in ileum homogenates.

Genes involved in NO biosynthesis. Genes involved in NO biosynthesis and signaling in the terminal ileum from rat pups at $\mathrm{P} 4$ are shown in Table 1 . Maternally fed groups were used 


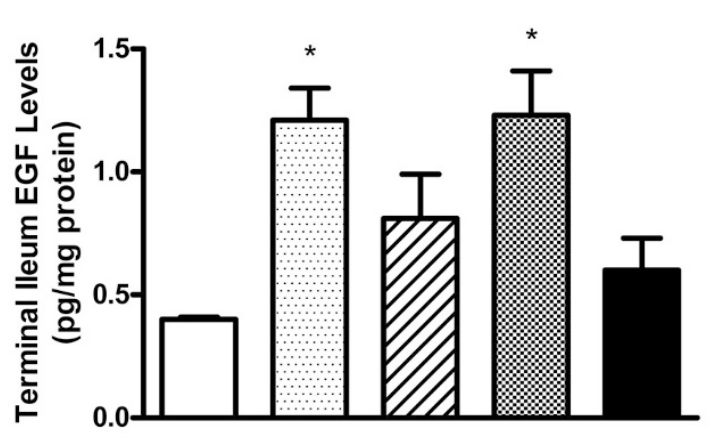

Figure 5. Mean EGF levels (ng/mg of protein) in terminal ileum homogenates. Groups are as described in Figure 1. Open bar, Mat-Fed; lightly shaded bar, Form-Fed; lined bar, Pre-Fed; heavily shaded bar, Pro-Fed; and solid bar, Syn-Fed. Only the pups that survived on the P4 were used for the analysis. ${ }^{*} p<0.05$ vs Mat-Fed.

Table 1. Fold change difference in mRNA expression of caveolin-1 and NOS isoforms in the terminal ileum of neonatal rats

\begin{tabular}{lcrrr}
\hline \multicolumn{1}{c}{ Genes } & Form-Fed & Pro-Fed & Pre-Fed & Syn-Fed \\
\hline eNOS & -79 & -115 & -73 & -217 \\
iNOS & 129 & 60 & 48 & 25 \\
nNOS & -93 & -55 & -22 & -73 \\
Caveolin-1 & -2097 & -432 & -1088 & -472 \\
Calmodulin-1 & -34 & -28 & -71 & -32 \\
\hline
\end{tabular}

Data are fold change difference in mRNA expression, with maternally fed group as baseline.

Form-Fed, formula fed with no supplementation; Pro-Fed, formula fed with probiotic supplementation; Pre-Fed, formula fed with prebiotic supplementation; Syn-Fed, formula fed with symbiotic supplementation.

as baseline. Compared with maternally fed group, caveolin-1, eNOS, nNOS, and calmodulin-1 expressions were downregulated in all the groups, whereas iNOS expression was up-regulated in all groups. Caveolin-1 was robustly downregulated in the unsupplemented formula group, and the effect was ameliorated with probiotics and synbiotics and, to a lesser degree, prebiotics.

Genes involved in oxidative stress response. Gene expression of SOD and GPX isoforms in the terminal ileum from rat pups at $\mathrm{P} 4$ are shown in Table 2. SOD-2 and -3, and copper chaperone for SOD genes are down-regulated in all formula groups. However, SOD-1 was up-regulated with prebiotics, probiotics, and synbiotics supplementation. GPX-2, -5, -6, and -7 , and catalase expressions were also down-regulated in all formula groups, but GPX-7 and catalase were substantially down-regulated in the unsupplemented formula group. Supplementation with probiotics preserved GPX-1, -3 , and -4 expressions, whereas prebiotics and synbiotics preserved GPX-3 expression. All supplements ameliorated GPX-7 and catalase expression.

Histopathology. Immediate postmortem performed on the animals lost each day demonstrated signs of discoloration, necrosis, and edema. None of the animals in the Mat-Fed group showed any symptoms consistent with NEC at the time of killing. A representative histologic sample from each group is shown in Figure 6. The terminal ileum architecture of the control, Mat-Fed group (Fig. 6A) showed multiple layers of abundant normal villi that appeared cuboidal and immature. No areas of hemorrhage, disruption, necrosis, or inflammation
Table 2. Fold change difference in mRNA expression of genes regulating oxidative stress in the terminal ileum of neonatal rats

\begin{tabular}{lrrrr}
\hline \multicolumn{1}{c}{ GENES } & Form-Fed & Pro-Fed & Pre-Fed & Syn-Fed \\
\hline SOD-1 & -15 & 11 & 12 & 15 \\
SOD-2 & -28 & -18 & -13 & -17 \\
SOD-3 & -101 & -29 & -7 & -19 \\
Copper chaperone & -2364 & -925 & -511 & -801 \\
$\quad$ for SOD & & & & \\
GPX-1 & -3 & 1 & -1 & -2 \\
GPX-2 & -22 & -6 & -2 & -8 \\
GPX-3 & -28 & 9 & 1 & 12 \\
GPX-4 & -29 & 3 & -4 & -7 \\
GPX-5 & -1546 & -455 & -335 & -542 \\
GPX-6 & -645 & -191 & -140 & -162 \\
GPX-7 & $-22,288$ & -120 & -80 & -174 \\
Catalase & -2096 & -634 & -634 & -2051 \\
\hline
\end{tabular}

Data are fold change difference in mRNA expression, with maternally fed group as baseline.

Form-Fed, formula fed with no supplementation; Pro-Fed, formula fed with probiotic supplementation; Pre-Fed, formula fed with prebiotic supplementation; Syn-Fed, formula fed with symbiotic supplementation; SOD, superoxide dismutase; GPX, glutathione peroxidase.

are seen. Mean circumference was $4066.4 \pm 23.80 \mu \mathrm{m}$, mean number of villi was $30.4 \pm 0.40$, and mean wall thickness was $28.3 \pm 2.70 \mu \mathrm{m}$. In the Form-Fed group (Fig. 6B), the terminal ileum architecture has the appearance of decreased number of villi that are disorganized and denuded with distinct areas of focal hemorrhage. Mean circumference was $2131.6 \pm$ $17.10 \mu \mathrm{m}(p<0.001$ versus Mat-Fed), mean number of villi was $24.0 \pm 0.40(p<0.01$ versus Mat-Fed $)$, and mean wall thickness was $42.1 \pm 0.80 \mu \mathrm{m}(p<0.001$ versus Mat-Fed). In the Pro-Fed group (Fig. 6C), the terminal ileum had many denuded villi, but they appeared to have maintained their integrity. There are no signs of hemorrhages, inflammation, or necrosis. Mean circumference was $2457.0 \pm 22.20 \mu \mathrm{m}(p<$ 0.001 versus Mat-Fed), mean number of villi was $28.8 \pm 0.40$ $(p<0.01$ versus Mat-Fed), and mean wall thickness was $26.8 \pm 1.70 \mu \mathrm{m}$. In the Pre-Fed group (Fig. $6 D$ ), the terminal ileum showed signs of severe injury associated with denuded villi, inflammation, and necrosis. Mean circumference was $3178.4 \pm 50.20 \mu \mathrm{m}(p<0.001$ versus Mat-Fed $)$, mean number of villi was $23.2 \pm 0.20$ ( $p<0.001$ versus Mat-Fed $)$, and mean wall thickness was $23.5 \pm 0.30 \mu \mathrm{m}(p<0.01$ versus Mat-Fed). In the Syn-Fed group (Fig. $6 E$ ), the terminal ileum appeared elongated and normal. There are no signs of hemorrhages, inflammation, or necrosis. Mean circumference was $2880.8 \pm 137.0 \mu \mathrm{m}(p<0.01$ versus Mat-Fed), mean number of villi was $28.0 \pm 0.50$ ( $p<0.01$ versus Mat-Fed), and mean wall thickness was $27.0 \pm 0.1 .50 \mu \mathrm{m}$ ( $p<0.01$ versus Mat-Fed). All images were taken from the surviving rats at $\mathrm{P} 4$.

\section{DISCUSSION}

This study tested the hypothesis that caveolin-1 is deficient in the terminal ileum of formula-fed neonatal rats and supplementation with $S$. Boulardii and/or FOS/GOS preserves normal terminal ileum caveolin-1 expression. Given the specific roles of IGF and EGF on fetal and neonatal gut epithelial cell proliferation $(21,22)$, and their relationship with caveolin-1 $(14,20)$, we examined the effects of enteral feeding with 

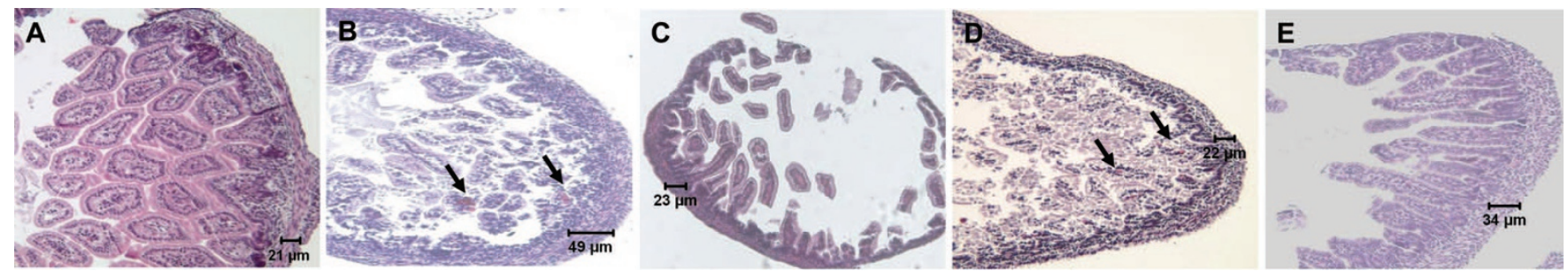

Figure 6. Representative histopathologic evaluation of the small intestine from each experimental group (hematoxylin and eosin stain, $\times 10$ magnification). The terminal ileum architecture of the control, Mat-Fed group $(A)$ showed multiple layers of abundant normal villi, which appeared cuboidal and immature. No areas of hemorrhage, disruption, necrosis, or inflammation are seen. In the Form-Fed group $(B)$, the terminal ileum appeared disorganized and denuded with distinct areas of focal hemorrhage (arrows). In the Pro-Fed group $(C)$, the terminal ileum had many denuded villi, but they appeared to have maintained their integrity. There are no signs of hemorrhages, inflammation, or necrosis. In the Pre-Fed group $(D)$, the terminal ileum showed signs of severe injury associated with denuded villi, inflammation, and necrosis. In the Syn-Fed group $(E)$, the terminal ileum appeared elongated and normal. There are no signs of hemorrhages, inflammation, or necrosis. All images were taken from the surviving rats at $\mathrm{P} 4$.

supplemented and unsupplemented formula on IGFs and EGF in the neonatal rat terminal ileum. Furthermore, considering the involvement of ROS scavengers on preserving $\mathrm{NO}$ and caveolin-1 signaling $(3,9)$, we determined whether supplementation increases the gene expression of SOD and GPX. The key findings of our study are as follows: 1) formula feeding with and without supplementation results in significant growth restriction compared with the maternally fed controls; 2) Despite suboptimal nutrition, IGF-I, IGF-2, and EGF, which play significant roles in intestinal mucosal repair, intestinal epithelial healing, regeneration, and permeability (23-27), were increased in the neonatal rat ileum; 3) caveolin-1, eNOS, and nNOS are simultaneously down-regulated with formula feeding, whereas iNOS is up-regulated. Supplementation did not reverse this effect; 4) SOD-2, SOD-3, and copper chaperone for SOD were down-regulated with formula feeding, an effect that remained sustained with supplementation. In contrast, SOD-1 was down-regulated with unsupplemented formula feeding and up-regulated with supplementation; and 5) GPX-1, -3, and -4 expressions were increased with $S$. Boulardii, whereas GPX-3 expression was preserved with FOS/GOS and combination of $S$. Boulardii and FOS/ GOS. All other GPX forms were down-regulated, particularly GPX-5, -6 , and -7 that were robustly decreased in the unsupplemented group. Catalase expression was down-regulated in all groups. Together, our findings may provide a probable mechanism for the benefits of supplementation on the incidence and severity of NEC that may involve induction of the antioxidant systems. Furthermore, our data may demonstrate a role for growth factors as local responders to intestinal injury.

The most compelling test of our hypothesis was demonstrated by the responses of the NOS isoforms and caveolin-1 in the formula-fed groups. We found that the constitutive forms of NOS, eNOS, and nNOS were down-regulated, whereas the inducible isoform, iNOS, was up-regulated in all formula and supplemented groups. However, although the increased expression of iNOS was less pronounced with supplementation, it was not totally protective. NO is an important inflammatory mediator that plays a key role in intestinal barrier failure seen in NEC. Sepsis and endotoxemia induce the formation of large amounts of iNOS that in turn has the capacity to produce large amounts of NO. Induction of iNOS and down-regulation of eNOS seen our study suggests exces- sive formation of NO that may in turn inactivate eNOS and nNOS. However, our results argue against excessive NO formation by iNOS, because we were unable to detect NOx levels in the terminal ileum homogenates. Nevertheless, our data showed that the constitutive isoforms of NOS are simultaneously down-regulated in the terminal ileum of formula-fed rats, suggesting that $\mathrm{NO}$ formation in the terminal ileum is predominantly derived from eNOS and/or nNOS. Our data support the findings of Gordon et al. (28) and suggest that simultaneous down-regulation of eNOS and nNOS, as well as caveolin-1, contribute to formula-induced terminal ileum inflammation in our model, thereby proving our hypothesis. However, supplementation did not appreciably reverse the effect.

To further support our hypothesis, we showed that supplementation up-regulated the genes that regulate oxidative stress. As previously mentioned, $\mathrm{NO}$ can be scavenged by $\mathrm{O}_{2}{ }^{-}$to produce ROS. SOD and NO compete for $\mathrm{O}_{2}{ }^{-}$, and therefore SOD is NO sparing. Our findings suggest that probiotics, prebiotics, and/or synbiotics are effective antioxidants and may be beneficial for combating the adverse effects of ROS. These studies may have implications for critically ill ELBW newborns where suboptimal enteral nutrition and inadequate formula feeding may result in significant postnatal growth restriction and predispose the immature gut to NEC. Our findings show an association of caveolin-1 and the constitutive isoforms of NOS in formula-induced bowel inflammation and suggest that the protective effect of probiotics, prebiotics, and synbiotics may involve in part mechanisms associated with oxidative stress and antioxidant responses.

Acknowledgments. We thank the staff of the Department of Laboratory Animal Research, State University of New York, Brooklyn, NY, for their assistance and technical support and the Pathology Department at State University of New York Downstate Medical Center, Brooklyn, NY, for their technical assistance.

\section{REFERENCES}

1. Srinivasan PS, Brandler MD, D'Souza A 2008 Necrotizing enterocolitis. Clin Perinatol 35:251-272

2. Levy RM, Prince JM, Billiar TR 2005 Nitric oxide: a clinical primer. Crit Care Med 33:S492-S495 
3. Alderton WK, Cooper CE, Knowles RG 2001 Nitric oxide synthases: structure, function and inhibition. Biochem J 357:593-615

4. Nathan C, Xie QW 1994 Nitric oxide synthases: roles, tolls, and controls. Cell 78:915-918

5. Tepperman BL, Brown JF, Whittle BJ 1993 Nitric oxide synthase induction and intestinal epithelial cell viability in rats. Am J Physiol 265:G214-G218

6. Ford H, Watkins S, Reblock K, Rowe M 1997 The role of inflammatory cytokines and nitric oxide in the pathogenesis of necrotizing enterocolitis. J Pediatr Surg $32: 275-282$

7. Beckman JS, Koppenol WH 1996 Nitric oxide, superoxide, and peroxynitrite: the good, the bad, and ugly. Am J Physiol 271:C1424-C1437

8. Upperman JS, Potoka D, Grishin A, Hackam D, Zamora R, Ford HR 2005 Mechanisms of nitric oxide-mediated intestinal barrier failure in necrotizing enterocolitis Semin Pediatr Surg 14:159-166

9. Bautista AP, Spitzer JJ 1994 Inhibition of nitric oxide formation in vivo enhances superoxide release by the perfused liver. Am J Physiol 266:G783-G788

10. Grande-García A, del Pozo MA 2008 Caveolin-1 in cell polarization and directional migration. Eur J Cell Biol 87:641-647

11. Hu G, Ye RD, Dinauer MC, Malik AB, Minshall RD 2008 Neutrophil caveolin-1 expression contributes to mechanism of lung inflammation and injury. Am J Physiol Lung Cell Mol Physiol 294:L178-L186

12. Salanueva IJ, Cerezo A, Guadamillas MC, del Pozo MA 2007 Integrin regulation of caveolin function. J Cell Mol Med 11:969-980

13. Warner BW, Warner BB 2005 Role of epidermal growth factor in the pathogenesis of neonatal necrotizing enterocolitis. Semin Pediatr Surg 14:175-180

14. Hirai C, Ichiba H, Saito M, Shintaku H, Yamano T, Kusuda S 2002 Trophic effect of multiple growth factors in amniotic fluid or human milk on cultured human fetal small intestinal cells. J Pediatr Gastroenterol Nutr 34:524-528

15. Maggi D, Biedi C, Segat D, Barbero D, Panetta D, Cordera R 2002 IGF-I induces caveolin 1 tyrosine phosphorylation and translocation in the lipid rafts. Biochem Biophys Res Commun 295:1085-1089

16. Lin HC, Hsu CH, Chen HL, Chung MY, Hsu JF, Lien RI, Tsao LY, Chen CH, Su BH 2008 Oral probiotics prevent necrotizing enterocolitis in very low birth weight preterm infants: a multicenter randomized controlled trial. Pediatrics 122:693-700

17. Khailova L, Dvorak K, Arganbright KM, Williams CS, Halpern MD, Dvorak B 2009 Changes in hepatic cell junctions structure during experimental necrotizing enterocolitis: effect of EGF treatment. Pediatr Res 66:140-144
18. Zani A, Ghionzoli M, Lauriti G, Cananzi M, Smith VV, Pierro A, De Coppi P, Eaton S 2010 Does intestinal permeability lead to organ failure in experimental necrotizing enterocolitis? Pediatr Surg Int 26:85-89

19. Liu Y, Zhu L, Fatheree NY, Liu X, Pacheco SE, Tatevian N, Rhoads JM 2009 Changes in intestinal toll-like receptors and cytokines precede histological injury in a rat model of necrotizing enterocolitis. Am J Physiol Gastrointest Liver Physiol 297:G442-G450

20. Repetto S, Salani B, Maggi D, Cordera R 2005 Insulin and IGF-I phosphorylate eNOS in HUVECs by a caveolin-1 dependent mechanism. Biochem Biophys Res Commun 337:849-852

21. Tremblay E, Chailler P, Ménard D 2001 Coordinated control of fetal gastric epithelial functions by insulin-like growth factors and their binding proteins. Endocrinology 142:1795-1803

22. Gordon PV 2009 Understanding intestinal vulnerability to perforation in the extremely low birth weight infant. Pediatr Res 65:138-144

23. Fruchtman S, Simmons JG, Michaylira CZ, Miller ME, Greenhalgh CJ, Ney DM, Lund PK 2005 Suppressor of cytokine signaling-2 modulates the fibrogenic actions of GH and IGF-I in intestinal mesenchymal cells. Am J Physiol Gastrointest Liver Physiol 289:G342-G350

24. Murali SG, Nelson DW, Draxler AK, Liu X, Ney DM 2005 Insulin-like growth factor-I (IGF-I) attenuates jejunal atrophy in association with increased expression of IGF-I binding protein-5 in parenterally fed mice. J Nutr 135:2553-2559

25. Huo H, Guo X, Hong S, Jiang M, Liu X, Liao K 2003 Lipid rafts/caveolae are essential for insulin-like growth factor-1 receptor signaling during 3T3-L1 preadipocyte differentiation induction. J Biol Chem 278:11561-11569

26. Matthews LC, Taggart MJ, Westwood M 2008 Modulation of caveolin-1 expression can affect signalling through the phosphatidylinositol 3-kinase/Akt pathway and cellular proliferation in response to insulin-like growth factor I. Endocrinology 149:5199-5208

27. Bennett WR, Crew TE, Slack JM, Ward A 2003 Structural-proliferative units and organ growth: effects of insulin-like growth factor 2 on the growth of colon and skin. Development 130:1079-1088

28. Gordon PV, Herman AC, Marcinkiewicz M, Gaston BM, Laubach VE, Aschner JL 2007 A neonatal mouse model of intestinal perforation: Investigating the harmful synergism between glucocorticoids and indomethacin. J Pediatr Gastroenterol Nutr 45:509-519 